\title{
Validación de Instrumento para Medir las Percepciones de los Docentes sobre el Diseño Universal para el Aprendizaje
}

\section{Instrument Validation to Measure Teachers' Perceptions of Universal Design for Learning}

\author{
Sergio Sánchez Fuentes *1 \\ David Jiménez Hernández ${ }^{2}$ \\ Patricia Sancho Requena ${ }^{3}$ \\ Irene Moreno-Medina ${ }^{1}$ \\ ${ }^{1}$ Universidad Autónoma de Madrid, España \\ ${ }^{2}$ Universidad Católica San Antonio de Murcia, España \\ ${ }^{3}$ Universidad de Valencia, España
}

\begin{abstract}
El diseño universal para el aprendizaje (DUA) ha sido estudiando en los últimos años como modelo para responder a la diversidad en el aula. Sin embargo, hay pocas investigaciones que proporcionen datos sobre su eficacia. El objetivo de este artículo es validar una herramienta que permita evaluar las percepciones basadas en DUA de los profesionales, a través del análisis factorial confirmatorio de una muestra de 230 profesionales. Para ello, una escala fue creada con 26 ítems: 9 ítems para el principio 1, 8 ítems para el principio 2 y 9 ítems para el principio 3. Los resultados muestran que la escala tenía una solución confirmatoria válida de tres factores, índices de confiabilidad satisfactorios y adecuada validez relacionada con los criterios. Los autores concluyen que se debe avanzar en la medición efectiva del DUA en la literatura científica, que el concepto está en constante evolución y, que, por tanto, la literatura científica y los trabajos académicos deben acompañar esta corriente basada en la inclusión y el derecho de todos a tener una educación en igualdad de oportunidades.
\end{abstract}

Descriptores: Diseño universal para el aprendizaje; Educación inclusiva; Profesorado; Evaluación.

The universal design for learning has been studied in recent years as a model for addressing diversity in the classroom. However, there are few scientific studies that provide data on their efficacy. The aim of this paper is to validate a tool which to evaluate the perceptions based on universal design for learning framework by professional, via confirmatory factor analysis, with a sample of 230 professionals. Reliability, factorial and criterial validity estimates are presented. A final scale was composite with 26 items: 9 items for principle 1, 8 items for principle 2 and 9 items for principle 3. Overall, the results shown that the scale had an adequate three-factor confirmatory solution, satisfying reliability indices, and adequate criterion-related validity. The authors conclude that we must advance in the effective measurement of UDL in the scientific literature, that the concept is constantly evolving and that, therefore, scientific literature and academic work should accompany this trend based on inclusion and the right of everyone to have an equal opportunity education.

Keywords: Universal design for learning; Inclusive education; Teacher; Assessment.

*Contacto: sergio.sanchezfuentes@uam.es

ISSN: 0718-7378

www.rinace.net/rlei/
Recibido: 06/11/2018

$1^{a}$ Evaluación: 18/12/2018

Aceptado: 12/03/2019 


\section{Revisión de la literatura}

En los últimos años, la educación inclusiva ha sido un eje común en los sistemas educativos con nuevos modelos basados en las diferencias (Armstrong, Armstrong y Barton, 2016) para dar respuesta a la diversidad de las aulas del siglo XXI. Entre los modelos y documentos estudiados más a fondo con una filosofía inclusiva, se podrían destacar los modelos que tienen el diseño universal como marco general de actuación (Sala et al., 2014). Incluso, dentro del propio modelo general, diversos espacios han sido creados con variaciones relacionadas con la aplicación de la filosofía del diseño universal a los espacios pedagógicos. De entre todos los marcos propuestos son, sin duda, 4 los modelos que han preponderado en la literatura académica: (a) Diseño Universal para el Aprendizaje (CAST, 2011); (b) el Diseño Universal para la Instrucción (Scott, McGuire y Foley, 2003); (c) el Diseño Instructivo Universal (Silver, Bourke y Strehorn, 1998); (d) el Diseño Universal en Educación (Burgstahler, 2009). Además, teniendo en cuenta los modelos expuestos anteriormente, en la actualidad, se han venido desarrollando diversos trabajos sobre las ventajas y experiencias de su implementación y mejora en el aprendizaje, pero se carece de datos a gran escala que puedan garantizar su funcionamiento en el aula (Sánchez et al., 2016) de manera generalizada. Los trabajos publicados, en la mayoría de los casos, son la descripción de experiencias y no estudios experimentales que permitan extrapolar conclusiones. Además, existe una mayor complejidad debido a que todos los modelos involucrados -Diseño Universal de Aprendizaje, Diseño Instruccional Universal, Diseño para la Instrucción Universal y Diseño Universal en Educación-(Ruiz et al., 2012) tienen un marco de referencia común, sin embargo, cada uno de estos conceptos se aplica de una manera diferente y con una filosofía y en una etapa educativa diferente (cuadro 1).

Por otro lado, se podría asegurar sin riesgo a equivocarse que en la última década es el diseño universal de aprendizaje (de ahora en adelante DUA) el modelo que se está estudiando de manera más extensa (Ok et al., 2017). Diferentes maneras de implementación están siendo analizadas por la comunidad científica (Ehlinger, 2017), pero en la mayoría de los casos, el DUA está siendo evaluado desde el punto de vista de la aplicabilidad de los procesos educativos en las etapas obligatorias de la educación (Ok et al., 2017). Numerosos estudios se han llevado a cabo sobre este campo (Dean, Lee-Post y Hapke, 2016; Hansen et al., 2016; Katz, 2015; Thomas et al., 2015; Smith y Harvey, 2014; Zamora-Musa et al., 2017). Todos ellos analizan la implementación del DUA en espacios no universitarios y describen los beneficios de este método, pero ninguno ofrece una muestra lo suficientemente amplia como para evidenciar conclusiones globales sobre el cambio en la metodología a aplicar en las aulas a nivel global.

Por otro lado, existen diversos estudios que describen experiencias sobre la aplicabilidad del diseño universal para el aprendizaje en el marco de la educación especial y en el campo de las personas con discapacidad. Smith y Lowrey (2017) planifican un modelo basado en el DUA para trabajar con personas con discapacidad intelectual.

Otros autores (Coyne et al., 2012) también trabajan para mejorar el diseño de los contenidos educativos con el paradigma DUA en niños con discapacidad intelectual. Y, aunque las personas con discapacidad intelectual son obviamente un grupo que se beneficia directamente al aplicar los principios de la DUA, otros grupos de personas con discapacidad también mejoran sus condiciones de aprendizaje si se aplica este modelo. 
Cuadro 1. Resumen de los principios que constituyen cada uno de los distintos enfoques sobre el paradigma del Diseño Universal aplicado a la educación

\begin{tabular}{|c|c|c|c|c|c|}
\hline TÉRMINOS & DUA & \multicolumn{2}{|c|}{ DIU } & DUI & DUE \\
\hline Centros & $\begin{array}{l}\text { Center for } \\
\text { Applied } \\
\text { Special } \\
\text { Technology } \\
\text { (CAST) }\end{array}$ & \multicolumn{2}{|c|}{$\begin{array}{l}\text { Georgian College; Brock } \\
\text { University; University of Queens; } \\
\text { University of Guelph } \\
\text { University of Minnestoa }\end{array}$} & $\begin{array}{l}\text { University of } \\
\text { Connecticut; } \\
\text { University } \\
\text { of } \\
\text { Wisconsin- } \\
\text { Milaukee }\end{array}$ & $\begin{array}{l}\text { University of } \\
\text { Washington }\end{array}$ \\
\hline $\begin{array}{c}\text { Principales } \\
\text { autores }\end{array}$ & $\begin{array}{l}\text { Rose y Meyer } \\
(2000,2002, \\
2006)\end{array}$ & \multicolumn{2}{|c|}{$\begin{array}{l}\text { Silver, Bouke y Strehorn (1998); } \\
\text { Bryson (2003); Palmer (2003) } \\
\text { Higbee (2003) }\end{array}$} & $\begin{array}{l}\text { Scott, Shaw y } \\
\text { McGuire } \\
(2003)\end{array}$ & $\begin{array}{l}\text { Burgstahler } \\
\text { (2002) }\end{array}$ \\
\hline Principios & $\begin{array}{l}\text { Proporcionar } \\
\text { múltiples } \\
\text { formas de } \\
\text { representación } \\
\text { Proporcionar } \\
\text { múltiples } \\
\text { formas de } \\
\text { acción y } \\
\text { expresión } \\
\text { Proporcionar } \\
\text { múltiples } \\
\text { formas de } \\
\text { participación }\end{array}$ & $\begin{array}{l}\text { Accesible e } \\
\text { imparcial en } \\
\text { todas las } \\
\text { partes } \\
\text { Consistente y } \\
\text { sencillo } \\
\text { Flexible en la } \\
\text { presentación, } \\
\text { participación y } \\
\text { uso } \\
\text { Explícitamente } \\
\text { presentado y } \\
\text { fácilmente } \\
\text { percibido } \\
\text { Proporciona un } \\
\text { entorno de } \\
\text { aprendizaje de } \\
\text { soporte } \\
\text { Minimiza el } \\
\text { esfuerzo y los } \\
\text { requerimientos } \\
\text { Asegura } \\
\text { espacios que se } \\
\text { acomodan }\end{array}$ & 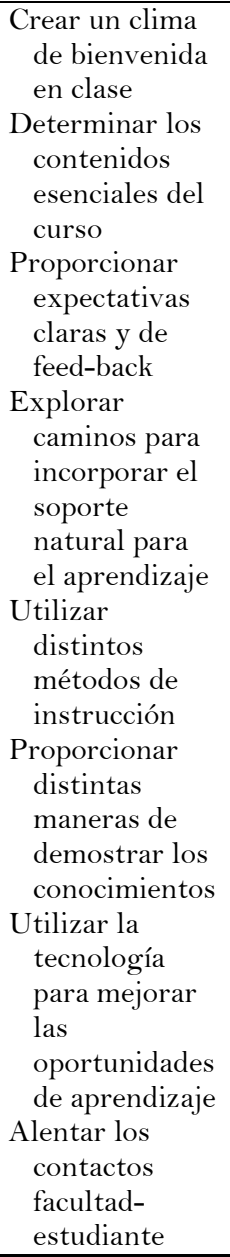 & $\begin{array}{l}\text { Uso } \\
\text { equitativo } \\
\text { Uso flexible } \\
\text { Uso intuitivo } \\
\text { y simple } \\
\text { Información } \\
\text { perceptible } \\
\text { Tolerancia al } \\
\text { error } \\
\text { Bajo esfuerzo } \\
\text { físico } \\
\text { Medidas y } \\
\text { espacios } \\
\text { adecuados } \\
\text { Comunidades } \\
\text { de } \\
\text { aprendizaje } \\
\text { Clima de } \\
\text { enseñanza } \\
\text { acogedor e } \\
\text { inclusivo }\end{array}$ & $\begin{array}{l}\text { Uso equitativo } \\
\text { Uso flexible } \\
\text { Uso intuitivo } \\
\text { y simple } \\
\text { Información } \\
\text { perceptible } \\
\text { Tolerancia al } \\
\text { error } \\
\text { Bajo esfuerzo } \\
\text { físico } \\
\text { Medidas y } \\
\text { espacios } \\
\text { adecuados }\end{array}$ \\
\hline
\end{tabular}

Nota: DuA: Diseño Universal para el Aprendizaje/Universal Design for Learning. DIU: Diseño Instruccional Universal/Universal Instruccional Design. DUI: Diseño Universal para la Instrucción/Universal Design for Instruction. DuE. Diseño Universal en Educación/Universal Design in Education.

Fuente: Elaboración propia a partir de Sala y otros (2014).

Por ejemplo, Cumming y Strnadová (2016) describen que los profesionales y los padres de personas con discapacidad del desarrollo mejoran sus percepciones sobre el uso del iPad si los contenidos han sido diseñados siguiendo los principios del DUA. Un aspecto estudiado y relacionado con la discapacidad es el uso de la tecnología de acuerdo con los tres principios del DUA (Alnahdi, 2014; Bryant, Rao y Ok, 2014; Mangiatordi, 2014). Sin embargo, en la mayoría de los casos, los autores se limitan a discutir los beneficios en el diseño y uso del DUA, pero no hay datos sobre su aplicación directa en los sistemas 
educativos. En menor medida, existen estudios que analizan la aplicación del DUA en campos tan diversos como la educación no formal (Ficarra y Chapin, 2016), en la educación de adultos (Chong, 2015) o en los modelos de bibliotecas (Webb y Hoover, 2015).

Finalmente, un campo explorado dentro de la aplicabilidad del marco del DUA es la educación superior. Más específicamente, en esta área se abordaron temas como el diseño de cursos on-line (Dell, Dell y Blackwell, 2015; Parker, 2012), revisiones teóricas (Díez y Sánchez, 2015) o estudios como la terapia ocupacional (Collins, 2014). Además, se han publicado artículos que analizan los diferentes enfoques basados en el diseño universal que describen el estado de la cuestión sobre la aplicabilidad del método en los espacios pedagógicos (Ruiz et al., 2012; Sala et al., 2014).

Sin embargo, existe escasa literatura que analice la estructura interna de los principios del DUA. El Center for Applied Special Technology (CAST) ha basado su marco teórico en la forma en que funciona el cerebro, pero no existen estudios rigurosos sobre la aplicabilidad concreta de la neurociencia basada en DUA que pueda transferirse al campo educativo. De hecho, las pautas 2.0 sobre el diseño universal para el aprendizaje tienen este modelo como marco de referencia (CAST, 2011).

Debido a la falta de estudios rigurosos sobre la aplicabilidad del DUA según la neurociencia y sus tres principios, sería necesario comenzar a trabajar en la dirección de obtener datos empíricos. Una forma de lograr este objetivo sería conocer las percepciones que involucran a los colectivos de estudiantes y profesores en el proceso de enseñanza y aprendizaje. Desde el punto de vista de los estudiantes de educación básica o educación primaria es complejo obtener datos válidos respondidos por los estudiantes, considerando que en la mayoría de los casos no son conscientes de las medidas basadas en DUA. Por lo tanto, una forma de obtener información adecuada es preguntar a los profesionales educativos cómo perciben las medidas basadas en el DUA y si están directamente relacionadas con los principios y directrices que CAST describe en las Pautas 2.0 del diseño universal para el aprendizaje.

De este modo, este trabajo tiene como objetivo principal conocer si las percepciones de los profesionales en el ámbito educativo sobre medidas basadas en las pautas 2.0 del diseño universal para el aprendizaje, realmente se correlacionan con los principios propuestos por CAST como modelo de inclusión educativa.

\section{Método}

\section{Participantes y procedimiento}

Los participantes del estudio fueron 230 futuros profesionales de la educación. Más concretamente, estudiantes de la modalidad presencial del Grado de Educación Infantil $(60 \%)$ y Primaria $(40 \%)$ de la Universidad San Antonio de Murcia. El 51,8\% provenían de la última etapa de estudios superiores (tercer y cuarto curso), y contaban con experiencia profesional. Las mujeres constituyeron la mayoría de la muestra $(75,2 \%)$. La edad media fue de 22,38 $(D T=4,06)$ con edades comprendidas entre 18 y 43 años.

La muestra forma parte de un estudio más amplio perteneciente a un proyecto universitario, cuyo objetivo fue investigar el conocimiento de los profesionales para desarrollar propuestas de capacitación adaptadas a las necesidades e intereses de la sociedad actual. Para llevar a cabo la investigación se optó por un diseño transversal y de 
encuesta, aplicando un muestreo no probabilístico de conveniencia. Siguiendo un procedimiento de autoinforme en lápiz y papel, se contó con la supervisión y el apoyo de algún miembro del equipo que resolvió las dudas que los participantes presentaron al respecto. La participación en el estudio fue voluntaria.

\section{Instrumentos}

Como se ha mencionado en el apartado anterior, este estudio forma parte de una investigación más amplia para detectar necesidades e intereses en el docente en el Espacio Europeo de Educación Superior (EEES). Es por esto que, el instrumento de medida constó de dos partes. En primer lugar, además de recoger información relativa a variables sociodemográficas, se incluyó un cuestionario diseñado ad-hoc para recabar información relativa al conocimiento de los futuros docentes en atención a la diversidad, TIC y métodos activos de enseñanza, así como las actuaciones que un maestro puede, y debe, llevar a cabo a este respecto. En segundo lugar, se recogió información relativa a la investigación de constructos psicológicos como son la Inteligencia Emocional, mediante el cuestionario TMMS-24 (Fernández-Berrocal, Extremera y Ramos, 2004) y la Autoestima, utilizando el cuestionario de Autoestima de Rosenberg (1965).

Para desarrollar el objetivo de este trabajo, se utilizaron escalas e indicadores psicosociales relacionados con la validación de las percepciones basadas en el Diseño Universal de Aprendizaje:

- Approaches to Teaching Inventory (Trigwell y Prosser, 2004). La ATI (Approaches to Teaching Inventory) se compone de 16 ítems que evalúan los enfoques de enseñanza empleados por el docente universitario, utilizando un formato de respuesta de tipo Likert. Elementos puntuados del 1 al $7(1=$ totalmente en desacuerdo, $4=$ totalmente de acuerdo), que representan dos estructuras factoriales. El alfa de Cronbach fue 0,702 en Cambio conceptual / centrado en el estudiante (CCCE/CCSF) y 0,729 en transmisión de información / centrado en el profesor (TICP/ITTF)

- Escala Trait Meta-Mood (Fernández-Berrocal, Extremera y Ramos, 2004). El alfa de Cronbach de 0,858 en percepción de inteligencia emocional, 0,849 de compresión de inteligencia emocional y 0,741 en la regulación de la inteligencia emocional.

\section{Análisis de datos}

Los análisis incluyeron estimaciones de consistencia interna para los ítems y la escala (es decir, alfa de Cronbach y homogeneidad de ítems). La validez relacionada con el criterio se evaluó relacionando la atención a la diversidad con la inteligencia emocional y los enfoques para el inventario de enseñanza. La validez constructiva (factorial) del ítem de cuestionario sobre percepciones basada en el DUA se evaluó con ESEM (modelos exploratorios de ecuaciones estructurales), a través del Análisis Factorial Confirmatorio (AFC), con método de estimación de mínimos cuadrados ponderados robustos (WLSMV: Weighted Least Squares Mean and Variance), el procedimiento de estimación recomendado no normal y ordinal (tipo Likert) (Finney y DiStefano, 2006).

La verosimilitud de cualquier AFC se ha evaluado usando varios criterios de ajuste (Hu y Bentler, 1999; Tanaka, 1993): (a) estadística de Chi-cuadrado (Kline, 2015; Ullman, 1996); (b) el índice de ajuste comparativo (CFI: Comparative Fit Index) de más de 0,90 (e, 
idealmente, más de 0,95, Hu y Bentler, 1999); (c) error cuadrático medio de aproximación -RMSEA: Root Mean Square Error of Approximation- (Steiger y Lind, 1980) de 0,05 o menos (el RMSEA utiliza errores de predicción y medición para evaluar el grado de coincidencia entre los modelos hipotéticos y los verdaderos); (d) el índice de bondad de ajuste (GFI: Goodness of Fit Index) y el TLI (índice Tucker-Lewis) como medidas de la proporción de varianza-covarianza explicadas para el modelo, con valores de más de 0,90 como indicativos de un ajuste adecuado (Hoyle y Panter, 1995); y (e) WRMR (raíz cuadrada media residual ponderada) con valores inferiores a 1 como indicativo de un ajuste adecuado ( $\mathrm{Yu}$ y Muthén, 2002). Todos los análisis estadísticos se realizaron con SPSS 17 y MPLUS 6 (para el AFC).

\section{Resultados}

\subsection{Análisis factorial confirmatorio}

Uno de los objetivos del estudio fue establecer la validez factorial de las percepciones basadas en el diseño universal para el aprendizaje. Una estructura teórica, de tres factores (cuadro 2) coincide con el marco DUA.

Cuadro 2. Cuestionario inicial sobre percepciones basadas en DUA

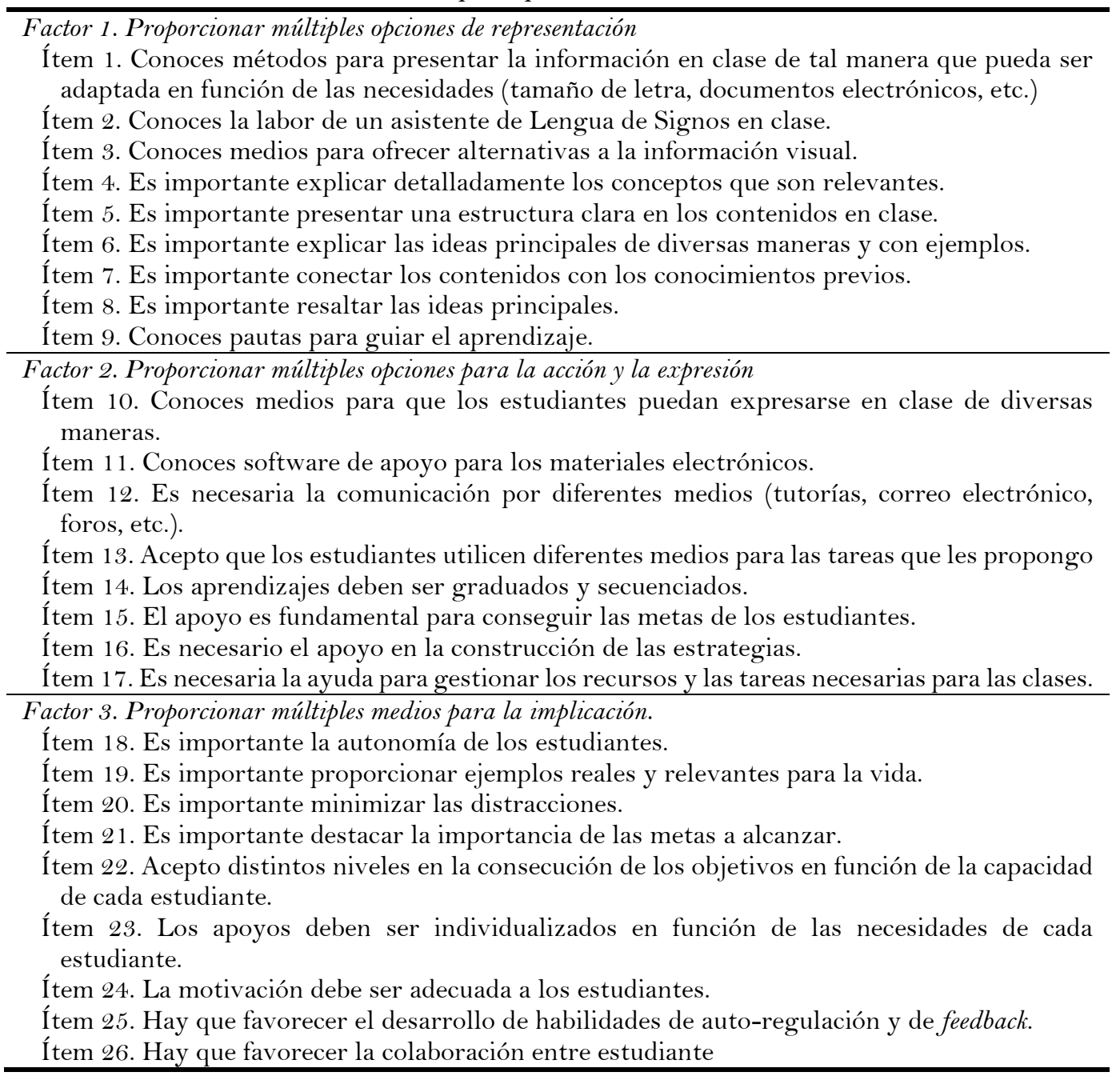

Fuente: Elaboración propia. 
Por lo tanto, se especificó, estimó y evaluó un análisis factorial confirmatorio con esta estructura a priori de tres factores. Este modelo teórico no mostró una buena coincidencia con los datos $\chi^{2}{ }^{296}=415,859, \mathrm{p}<0,001, \mathrm{CFI}=0,909$, TLI $=0,900$, RMSEA $=0,059[0,045$ $-0,072]$, WRMR $=1,006$. Dado este resultado, se realizó una depuración de elementos no significativos (ítems 1, 2, 7, 9, 25 y 26). Los índices de ajuste general apoyaron principalmente el nuevo modelo, con estructura de tres factores de la escala: el chicuadrado fue de 205,840, con 167 grados de libertad ( $p<0,05$ ), el CFI fue de 969, TLI fue de 0,965, RMSEA fue de 0,045 [-0,018-,064], y WRMR fue 0,797. En conjunto, los índices evaluaron el modelo como una representación adecuada de los datos observados. El cuestionario final está compuesto por 20 ítems.

Por otro lado, un examen detallado de las cargas de los factores arrojó una idea del ajuste analítico del modelo, complementando la información de ajuste general. Todos los indicadores puntuaron significativamente alto en el correspondiente factor de hipótesis, lo que apoya la idoneidad del modelo de tres factores.

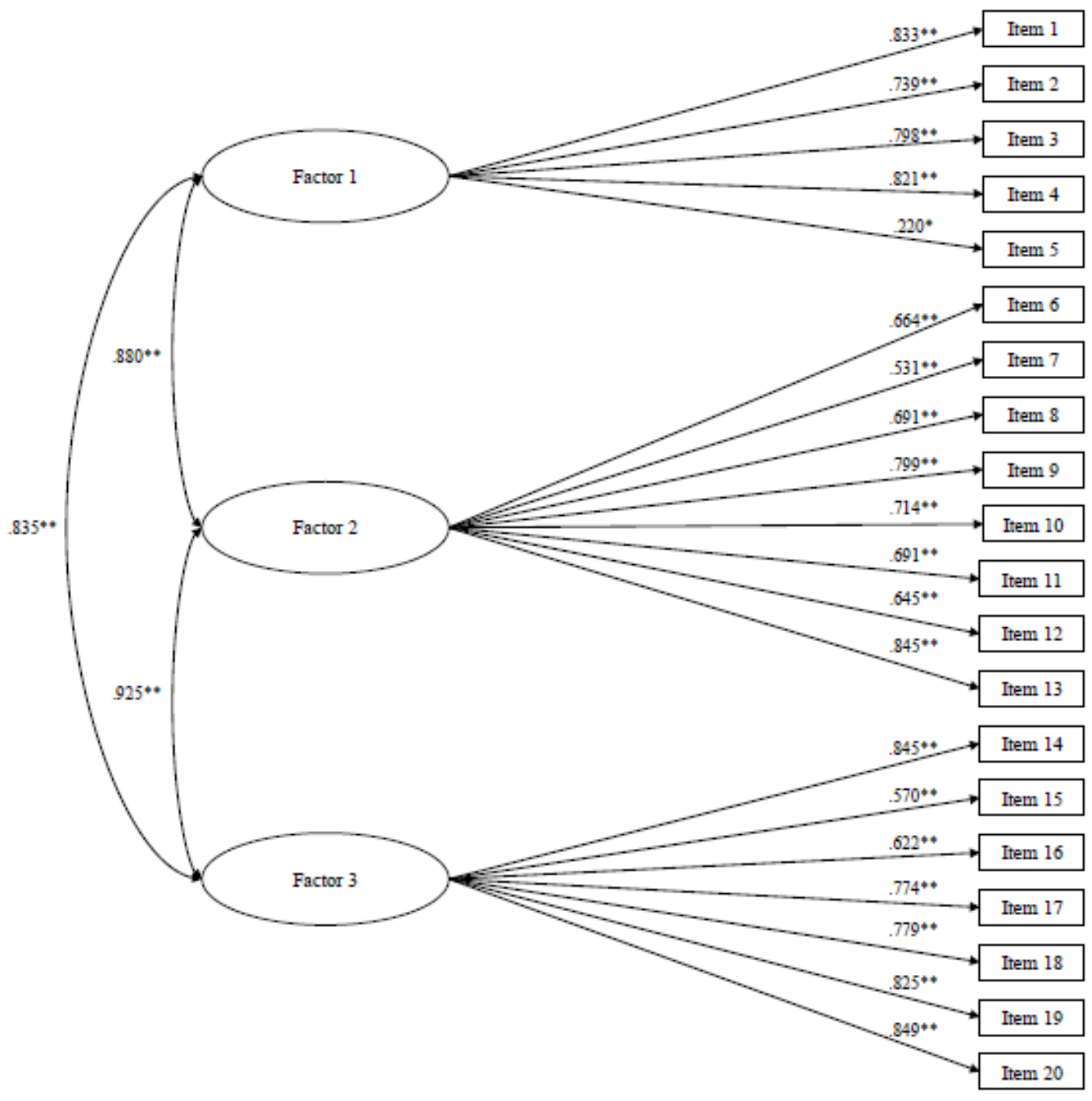

Figura 1. Saturación factorial estandarizado para el factor de satisfacción de vida Fuente: Elaboración propia. 
Como se muestra en la figura 1, las puntuaciones factoriales estandarizadas para las percepciones basadas en DUA estuvieron dentro de un mínimo de 0,220 (ítem 8, "Es importante resaltar las ideas principales") y un máximo de 0,849 (ítem 24, "La motivación debe ser apropiada para los estudiantes"). Todas las saturaciones factoriales estuvieron muy por encima de los valores considerados indicativos de una adecuada consistencia con el factor a priori.

\subsection{Consistencia interna}

El cuadro 3 presenta los promedios, las desviaciones estándar y la correlación corregida de cada ítem con la puntuación total de la dimensión correspondiente sin el ítem mismo, como una medida de confiabilidad. Como se puede ver, las medias están cerca del punto medio de la escala de respuesta $(2,5)$. En general, la homogeneidad de los ítems es adecuada, con un mínimo de 0,129 para el ítem 1 y un máximo de 0,605 para el ítem 12. La media de las correlaciones ítem-dimensión es 0,417, con una desviación estándar de 0,124 . Además, se han calculado las alfas de cada una de las dimensiones de atención a la diversidad, con valores de .634 en los múltiples medios de representación (Factor 1 de DUA), 0,730 en los múltiples medios de acción y expresión (Factor 2 de DUA) y 0,760 en múltiples medios de participación o interés (Factor 3 de DUA).

Cuadro 3. Significado, Desviación estándar, homogeneidad de ítem y Alpha si el ítem es borrado para los cinco ítems de la Percepción de acciones basadas en el Diseño Universal para el Aprendizaje

\begin{tabular}{|c|c|c|c|c|}
\hline ÍTEM PABUDL & SIGNIFICADO & $\begin{array}{c}\text { DESVIACIÓN } \\
\text { ESTÁNDAR }\end{array}$ & $\begin{array}{l}\text { HOMOG. } \\
\text { DE ÍTEM }\end{array}$ & $\begin{array}{l}\text { ALPHA SI } \\
\text { EL ÍTEM } \\
\text { ES } \\
\text { BORRADO }\end{array}$ \\
\hline \multicolumn{5}{|c|}{ Factor 1: Proporcionar múltiples opciones de representación } \\
\hline $\begin{array}{l}\text { Conoces medios para ofrecer } \\
\text { alternativas a la información } \\
\text { visual }\end{array}$ & 2,518 & 0,921 & 0,129 & 0,637 \\
\hline $\begin{array}{l}\text { Es importante explicar } \\
\text { detalladamente los conceptos que } \\
\text { son relevantes }\end{array}$ & 2,876 & 0,819 & 0,306 & 0,515 \\
\hline $\begin{array}{l}\text { Es importante presentar una } \\
\text { estructura clara en los contenidos } \\
\text { en clase }\end{array}$ & 3,665 & 0,578 & 0,448 & 0,451 \\
\hline $\begin{array}{l}\text { Es importante explicar las ideas } \\
\text { principales de diversas maneras y } \\
\text { con ejemplos }\end{array}$ & 3,775 & 0,498 & 0,392 & 0,484 \\
\hline $\begin{array}{l}\text { Es importante resaltar las ideas } \\
\text { principales }\end{array}$ & 3,821 & 0,429 & 0,399 & 0,492 \\
\hline \multicolumn{5}{|c|}{ Factor 2: Proporcionar múltiples opciones para la acción y la expresión } \\
\hline $\begin{array}{l}\text { Conoces medios para que los } \\
\text { estudiantes puedan expresarse en } \\
\text { clase de diversas maneras }\end{array}$ & 2,966 & 0,667 & 0,282 & 0,731 \\
\hline $\begin{array}{l}\text { Conoces software de apoyo para } \\
\text { los materiales electrónicos }\end{array}$ & 2,698 & 0,790 & 0,173 & 0,764 \\
\hline $\begin{array}{l}\text { Es necesaria la comunicación por } \\
\text { diferentes medios -tutorías, } \\
\text { correo electrónico, foros, etc.- }\end{array}$ & 3,574 & 0,608 & 0,397 & 0,707 \\
\hline $\begin{array}{l}\text { Acepto que los estudiantes utilicen } \\
\text { diferentes medios para las tareas } \\
\text { que les propongo }\end{array}$ & 3,502 & 0,605 & 0,448 & 0,697 \\
\hline $\begin{array}{l}\text { Los aprendizajes deben ser } \\
\text { graduados y secuenciados }\end{array}$ & 3,559 & 0,625 & 0,510 & 0,684 \\
\hline
\end{tabular}


El apoyo es fundamental para

conseguir las metas de los

3,684

0,559

0,560

0,678

estudiantes

Es necesario el apoyo en la

construcción de las estrategias

3,540

0,604

0,605

0,666

Es necesaria la ayuda para

gestionar los recursos y las tareas

3,483

0,605

0,528

0,682 necesarias para las clases

\begin{tabular}{|c|c|c|c|c|}
\hline \multicolumn{5}{|c|}{ Factor 3: Proporcionar múltiples medios para la implicación } \\
\hline $\begin{array}{l}\text { Es importante la autonomía de los } \\
\text { estudiantes }\end{array}$ & 3,603 & 0,6082 & 0,425 & 0,710 \\
\hline $\begin{array}{l}\text { Es importante proporcionar } \\
\text { ejemplos reales y relevantes para } \\
\text { la vida }\end{array}$ & 3,732 & 0,5114 & 0,599 & 0,674 \\
\hline $\begin{array}{l}\text { Es importante minimizar las } \\
\text { distracciones }\end{array}$ & 3,562 & O,6063 & 0,399 & 0,716 \\
\hline $\begin{array}{l}\text { Es importante destacar la } \\
\text { importancia de las metas a } \\
\text { alcanzar }\end{array}$ & 3,543 & 0,5925 & 0,376 & 0,721 \\
\hline $\begin{array}{l}\text { Acepto distintos niveles en la } \\
\text { consecución de los objetivos en } \\
\text { función de la capacidad de cada } \\
\text { estudiante }\end{array}$ & 3,506 & 0,6018 & 0,431 & 0,708 \\
\hline $\begin{array}{l}\text { Los apoyos deben ser } \\
\text { individualizados en función de las } \\
\text { necesidades de cada estudiante }\end{array}$ & 3,589 & 0,6180 & 0,446 & 0,705 \\
\hline $\begin{array}{l}\text { La motivación debe ser adecuada a } \\
\text { los estudiantes }\end{array}$ & 3,741 & 0,5161 & 0,495 & 0,695 \\
\hline
\end{tabular}

Fuente: Elaboración propia.

\subsection{Validación}

La validez relacionada con el criterio se estableció al correlacionar la atención a la diversidad con constructos teóricamente vinculados a este factor en la literatura. Específicamente, las percepciones basadas en el DUA se relacionaron con la inteligencia emocional, los enfoques de enseñanza y la autoestima. Excepto ADF 1 con compresión de inteligencia emocional y $\mathrm{ADF} 2$ con regulación de inteligencia emocional, donde se encontró un patrón consistente de relaciones positivas entre cada una de estas dimensiones y los tres factores del DUA, que es consistente con los resultados esperados (cuadro 4).

Cuadro 4. Correlaciones del PABUDL con una medida de inteligencia emocional, enfoques de enseñanza y autoestima

\begin{tabular}{lcccccccc}
\hline & UDLF1 & UDLF2 & UDLF3 & EIP & EIC & EIR & CCSF & ITTF \\
\hline UDLF 1 & 1 & & & & & & & \\
UDLF2 & $0,598^{* *}$ & 1 & & & & & & \\
UDLF3 & $0,592^{* *}$ & $0,639^{* *}$ & 1 & & & & & \\
EIP & $0,136^{*}$ & $0,214^{* *}$ & $0,216^{* *}$ & 1 & & & & \\
EIC & 0,113 & $0,147^{*}$ & $0,194^{* *}$ & 0,054 & 1 & & & \\
EIR & $0,135^{*}$ & 0,120 & $0,201^{* *}$ & $-0,003$ & $0,576^{* *}$ & 1 & & \\
CCSF & $0,387^{* *}$ & $0,555^{* *}$ & $0,561^{* *}$ & $0,198^{* *}$ & $0,210^{* *}$ & $0,240^{* *}$ & 1 & \\
ITTF & $0,190^{* *}$ & $0,281^{* *}$ & $0,351^{* *}$ & $0,159^{*}$ & 0,078 & 0,088 & $0,561^{* *}$ & 1 \\
Autoestima & $0,168^{*}$ & $0,222^{* *}$ & $0,162^{*}$ & $-0,113$ & $0,365^{* *}$ & $0,401^{* *}$ & $0,170^{*}$ & 0,003 \\
\hline
\end{tabular}

Nota: $* *$ $p<0,01 ; * \mathrm{p}<0,05$.

Fuente: Elaboración propia. 


\section{Discusión y conclusiones}

A través de la validación de este cuestionario, se ha intentado presentar una herramienta para evaluar las percepciones del diseño universal para el aprendizaje por profesionales del ámbito educativo. Como se puede ver en los resultados, la herramienta sí se ajusta al modelo teórico basado en el diseño universal para el aprendizaje. Sin embargo, hay ciertos aspectos que deben destacarse.

Primero, se debe resaltar que la relación entre las pautas 2.0 y las percepciones no es exactamente equitativa. Teniendo en cuenta que cada ítem tenía una correspondencia y relación directa con el punto de control de las pautas, se esperaba por parte de los autores que los ítems encajaran directamente con el modelo presentado por CAST. Sin embargo, los datos muestran que solo unos pocos elementos constituyen y se relacionan con el factor $1 \mathrm{o}$ el principio 1 del DUA. Acciones como ofrecer flexibilidad, tener un intérprete de lenguaje de signos en clase o conectar el aprendizaje y resaltar las ideas principales no están presentes en dicho factor, siendo estos aspectos fundamentales en la manera en que los profesores proporcionamos la información a nuestros estudiantes por diferentes vías. Por lo tanto, desde el punto de vista de los docentes, como primer agente educativo y como proveedores de información a los estudiantes no parece que las percepciones sobre cómo realizamos nuestro trabajo estén relacionadas directamente con lo expuesto por el paradigma del DUA en su principio 1.

Sin embargo, los resultados han arrojado mejores puntaciones para el factor 2 o el principio 2 del DUA. Un factor inicial con 8 ítems ha resultado ser fiel al modelo propuesto por CAST en el DUA. Este hecho es significativo porque el cuestionario validado explora las percepciones de los docentes y no de los estudiantes. Sin embargo, el factor relacionado con el alumno se ajusta más adecuadamente al cuestionario inicial que el factor relacionado con el docente. Es decir, los maestros perciben mejor las opciones que deberían darles a los estudiantes a la hora de permitir expresar lo que ellos saben en las tareas exigidas por los profesores. Es, por ello, que si los profesionales somos conscientes de que debemos otorgar flexibilidad en las tareas que demandamos a nuestros estudiantes conseguiremos estudiantes más motivados y dispuesto a aprender. Trabajos como el presentado por Rose y otros (2006) donde los estudiantes tuvieron mayores oportunidades para poder responder a lo que se les demandaba, fueron, posteriormente, puntuados como mejores formaciones por parte de los estudiantes, Es decir, una mayor flexibilidad a la hora de entregar o presentar los materiales, hace que las actividades metacognitivas de los estudiantes mejoren sobre su propio aprendizaje.

Por otro lado, el factor 3, relacionado con la participación y el compromiso de los estudiantes por su propio aprendizaje, se ajusta a un modelo que considera todo lo descrito en las directrices 2.0 sobre el DUA, menos aquellos ítems relacionados con la autorregulación y la colaboración. En términos generales, los profesionales describen que conocen las opciones que deberían permitir a los estudiantes mejorar su propio aprendizaje. En parte, podría destacarse como lógico este resultado, ya que los propios docentes no pueden tener acceso a las opciones de autorregulación y control de las emociones de cada uno de sus estudiantes (Borgobello, Sartori y Sanjurjo, 2018)

Por lo tanto, a tenor de los resultados hallados, los autores consideran la herramienta como válida como un primer instrumento para medir las percepciones de los docentes sobre medidas basadas en el diseño universal para el aprendizaje. Esto es debido en parte 
a un ajuste en gran medida a lo que CAST estableció en las pautas 2.0 del DUA. Una de las fortalezas del presente documento es que la herramienta es una medida para evaluar las percepciones del modelo de diseño universal de aprendizaje por parte de profesionales que tienen propiedades psicométricas, validez y confiabilidad sólidas. La escala podría tener una utilidad potencial tanto en la práctica educativa como en la investigación en diversos contextos educativos en lengua castellana o española. La segunda fortaleza de este trabajo es que el instrumento supone la primera validación con análisis confirmatorio del modelo DUA aplicado directamente en el aula. Son pocos los trabajos que tienen como objetivo principal explorar el modelo teórico del diseño universal de aprendizaje (Ruiz et al., 2012; Sánchez et al., 2016): y aún más, aquellos que han logrado describir una herramienta para la evaluación de DUA por parte de profesionales. La importancia de contar con herramientas que nos permitan evaluar el grado de mejora del aprendizaje de los estudiantes debería ser un área de estudio prioritaria en educación (Shepard, 2000).

Además, hay que añadir que debido a la evolución que los conceptos van sufriendo con el paso del tiempo gracias al estudio por parte de la comunidad científica, el propio diseño universal para el aprendizaje está en constante desarrollo. Esto, que supone un avance al considerar los aspectos educativos como una ciencia basada en la evidencia, puede también suponer un impedimento si no se atiende de manera urgente las demandas que las investigaciones van desarrollando (CAST, 2018). Un ejemplo de la importancia de lo anteriormente expuesto es la próxima publicación de las pautas sobre el diseño universal para el aprendizaje. Y, es que a pesar que el modelo presentado en las pautas 2.0 lleva vigente desde 2011 , nulos o escasos han sido los trabajos que se han focalizado en trasladar el modelo planteado en dicho documento a una herramienta que pueda ser utilizada por los investigadores para medir las percepciones de los profesionales educativos respecto de la materia aquí tratada. Un ejemplo de una apuesta fuerte por este modelo es Chile, que no solo ha desarrollado normativa al respecto, sino que los proyectos basados en el paradigma del diseño universal para el aprendizaje están siendo desarrollados por numerosos investigadores en los últimos años.

Sin embargo, el estudio tiene algunas limitaciones. No se pudieron proporcionar estimaciones de la fiabilidad test-retest en el estudio actual, ya que la muestra solo se evaluó una vez. Dicha información podría brindar información adicional sobre la confiabilidad de la escala.

Como líneas futuras de investigación, los estudios con datos interculturales serían de interés, validando el modelo de percepciones basadas en el DUA en muestras de diferentes países y de una amplia gama de poblaciones relacionadas con el área educativa.

\section{Referencias}

Alnahdi, G. (2014). Assistive technology in special education and the universal design for learning. Turkish Online Journal of Educational Technology, 13(2), 18-23.

Armstrong, F., Armstrong, D. y Barton, L. (2016). Inclusive education: Policy, contexts and comparative perspectives. Londres: Routledge. https://doi.org/10.4324/9780203065075

Borgobello, A., Sartori, M. y Sanjurjo, L. O. (2018). Concepciones de docentes sobre los estudiantes y sus prácticas pedagógicas. Educación y Educadores, 21(1), 27-48.

Bryant, B. R., Rao, K. y Ok, M. W. (2016). Universal design for learning and assistive technology: Promising developments. En P. Zaphiris y C. Siang (Eds.), Human-computer interaction: 
Concepts, methodologies, tools, and applications (pp. 567-582). Londres: IGI Global. https://doi.org/10.4018/978-1-4666-8789-9.ch025

Burgstahler, S. (2009). Universal design in education: Principles and applications. Recuperado de https://www.washington.edu/doit/sites/default/files/atoms/files/Universal-DesignEducation-Principles-Applications.pdf

Center for Applied Special Technology. (2011). Universal design for learning guidelines version 2.0. Wakefield, MA: CAST.

Center for Applied Special Technology. (2018). Universal design for learning guidelines version 2.2. Wakefield, MA: CAST.

Chong, S. (2015). Unpacking the digital backpack for the adult learner. A universal design for learning approach. En J. Lam, K. Keung, S. Cheung, T. Wong, K. Li y F. Wang (Comps.), Proceedings of the international conference on technology in education (pp. 10-22). Berlin: Springer.

Collins, B. (2014). Universal design for learning: What occupational therapy can contribute. Occupational Therapy Now, 16(5), 22-23.

Coyne, P., Pisha, B., Dalton, B., Zeph, L. A. y Smith, N. C. (2012). Literacy by design: A universal design for learning approach for students with significant intellectual disabilities. Remedial and Special Education, 33(3), 162-172. https://doi.org/10.1177/0741932510381651

Cumming, T. M. y Strnadová, I. (2016). Parents' and teachers' perspectives on using iPads with students with developmental disabilities. En K. Miesenberger y G. Kouroupetroglou (Eds.), Proceedings of the international conference on computers helping people with special needs (pp. 217222). Cham: Springer. https://doi.org/10.1007/978-3-319-41267-2_29

Dean, T., Lee-Post, A. y Hapke, H. (2016). Universal design for learning in teaching large lecture classes. Journal of Marketing Education, 39(1), 5-16. https://doi.org/10.1177/0273475316662104

Dell, C. A., Dell, T. F. y Blackwell, T. L. (2015). Applying universal design for learning in online courses: Pedagogical and practical considerations. Journal of Educators Online, 13(2), 166192 .

Díez, E. y Sánchez, S. (2015). Universal design for learning as a teaching method in order to meet the need for diversity in universities. Aula Abierta, 43(2), 87-93.

https://doi.org/10.17811/rifie.43.2015.87-93

Ehlinger, E. (2017). Expanding notions of access: Opportunities and future directions for universal design. En H., Alphin, J. Lavine y R. Chan (Eds.), Disability and equity in higher education accessibility (pp. 204-221). Londres: IGI Global.

Fernández-Berrocal, P., Extremera, N. y Ramos, N. (2004). Validity and reliability of the Spanish modified version of the trait meta-mood scale. Psychological Reports, 94(3), 751-755. https://doi.org/10.2466/pro.94.3.751-755

Ficarra, L. R. y Chapin, D. A. (2016). Reconceptualizing universal design for learning (UDL) as learning technology in non-formal education. En J. Keengwe y P. Bull (Coords.), Handbook of research on transformative digital content and learning technologies (pp. 81-102). Londres: IGI Global.

Finney, S. J. y DiStefano, C. (2006). Non-normal and categorical data in SEM. En G. Hancock y R. Mueller (Eds.), Structural equation modelling: A second course (pp. 269-314). Greenwich, CO: Information Age Publishing. 
Hansen, A. K., Hansen, E. R., Dwyer, H. A., Harlow, D. B. y Franklin, D. (2016). Differentiating for diversity: Using universal design for learning in computer science education. En C. Alphonce (Comp.), Proceedings of the 47th ACM technical symposium on computing science education (pp. 376-381). Memphis, TN: ACM.

Hoyle, R. H. y Panter, A. T. (1995). Writing about structural equation models. En R. H. Hoyle (Ed.), Structural equation modeling: Concepts, issues, and applications (pp. 159-176). Thousand Oaks, CA: Sage.

Hu, L. y Bentler, P. M. (1999). Cut-off criteria for fit indexes in covariance structure analysis: Conventional criteria versus new alternatives. Structural Equation Modeling, 6, 1-55. https://doi.org/10.1080/10705519909540118

Katz, J. (2015). Implementing the three blocks model of universal design for learning: Effects on teachers' self-efficacy, stress, and job satisfaction in inclusive classrooms K-12. International Journal of Inclusive Education, 19(1), 1-20. https://doi.org/10.1080/13603116.2014.881569

Kline, R. B. (2015). Principles and practice of structural equation modeling. Nueva York, NY: Guilford.

Mangiatordi, A. (2014). Using universal design for learning guidelines to evaluate a computer assisted note taking software solution. En M. Gennari (Coord.), Methodologies and intelligent systems for technology enhanced learning (pp. 157-164). Cham: Springer. https://doi.org/10.1007/978-3-319-07698-0_20

Ok, M. W., Rao, K., Bryant, B. R. y McDougall, D. (2017). Universal design for learning in pre-k to grade 12 classrooms: A systematic review of research. Exceptionality, 25(2), 116-138. https://doi.org/10.1080/09362835.2016.1196450

Parker, H. (2012). Learning starts with design: Using universal design for learning (UDL) in higher education course redesign. En F. S. Miller (Ed.), Transforming learning environments: Strategies to shape the next generation (pp. 109-136). Bingley: Emerald Group Publishing Limited. https://doi.org/10.1108/S1479-3660(2012)0000016009

Rose, D. H., Harbour, W. S., Johnston, C. S., Daley, S. G. y Abarbanell, L. (2006). Universal design for learning in postsecondary education: Reflections on principles and their application. Journal of Postsecondary Education and Disability, 19(2), 135-151.

Rosenberg, M. (1965). Society and the adolescent self-image. Princeton, NJ: Princeton. https://doi.org/10.1515/9781400876136

Ruiz, R., Solé, L., Echeita, G., Sala, I. y Datsira, M. (2012). El principio del “universal design”: concepto y desarrollos en la enseñanza superior. Revista de Educación, 359, 413-430.

Sala, I., Sánchez, S., Giné, C. y Díez, E. (2014). Análisis de los distintos enfoques del paradigma del diseño universal aplicado a la educación. Revista Latinoamericana de Educación Inclusiva, 8(1), 143-152.

Sánchez, S., Castro, L., Casas, J. A. y Vallejos, V. (2016). Análisis factorial de las percepciones docentes sobre diseño universal de aprendizaje. Revista Latinoamericana de Educación Inclusiva, 1O(2), 135-149. https://doi.org/10.4067/So7 18-73782016000200009

Scott, S. S., McGuire, J. M. y Foley, T. E. (2003). Universal design for instruction: A framework for anticipating and responding to disability and other diverse learning needs in the college classroom. Equity E'Excellence in Education, 36(1), 40-49.

https://doi.org/10.1080/10665680303502

Shepard, L. A. (2000). The role of assessment in a learning culture. Educational Researcher, 29(7), 4-14. https://doi.org/10.3102/0013189X029007004 
Silver, P., Bourke, A. y Strehorn, K. C. (1998). Universal instructional design in higher education: An approach for inclusion. Equity \& Excellence, 31(2), 47-51. https://doi.org/10.1080/1066568980310206

Smith, S. J. y Harvey, E. E. (2014). K-12 online lesson alignment to the principles of universal design for learning: The khan academy. Open Learning, 29(3), 222-242. https://doi.org/10.1080/02680513.2014.992402

Smith, S. J. y Lowrey, K. A. (2017). Applying the universal design for learning framework for individuals with intellectual disability: The future must be now. Intellectual and Developmental Disabilities, 55(1), 48-51. https://doi.org/10.1352/1934-9556-55.1.48

Steiger, J. H. y Lind, C. (1980). Statistically based tests for the number of common factors. Comunicación presentada en el Annual Meeting of the Psychometric Society. The University of Iowa.

Tanaka, J. S. (1993). Multifaceted conceptions of fit in structural equation models. En K. A. Bollen (Ed.), Testing structural equation models (pp. 10-39). Newbury Park, CA: Sage.

Thomas, C. N., Van Garderen, D., Scheuermann, A. y Lee, E. J. (2015). Applying a universal design for learning framework to mediate the language demands of mathematics. Reading and Writing Quarterly, 31 (3), 207-234. https://doi.org/10.1080/10573569.2015.1030988

Trigwell, K. y Prosser, M. (2004). Development and use of the approaches to teaching inventory. Educational Psychology Review, 16(4), 409-424. https://doi.org/10.1007/s 10648-004-0007-9

Ullman, J. B. (1996). Structural equation modeling. En B. Tabachnick y L. Fidell (Eds.), Using multivariate statistics (pp. 709-812). Nueva York, NY: Harper Collins.

Webb, K. K. y Hoover, J. (2015). Universal design for learning (UDL) in the academic library: A methodology for mapping multiple means of representation in library tutorials. College and Research Libraries, 76(4), 537-553. https://doi.org/10.5860/crl.76.4.537

Yu, C. Y. y Muthen, B. (abril, 2002). Evaluation of model fit indices for latent variable models with categorical and continuous outcomes. Comunicación presentada en el Annual meeting of the American Educational Research Association. University of New Orleans.

Zamora-Musa, R., Vélez, J., Paez-Logreira, H., Coba, J., Cano-Cano, C. y Palmera, O. M. (2017). Implementation of an open educational resource through the model of universal design for learning taking into account competency-based assessment and the individual needs of students. Espacios, 38(5), 3-12.

\section{Breve CV de los autores}

\section{Sergio Sánchez Fuentes}

Doctor en Educación por la Universidad de Salamanca. Es profesor ayudante doctor y subdirector del Departamento de Psicología Evolutiva y de la Educación de la Universidad Autónoma de Madrid. Trabaja en proyectos nacionales, europeos e internacionales sobre el análisis de la situación de las personas con discapacidad en la educación, con especial hincapié en el análisis del paradigma del diseño universal de aprendizaje. Actualmente pertenece a diferentes grupos de investigación en España. Colabora com diversas universidades en países de Europa, Asia y América. Ha formado a profesionales del ámbito educativo en numerosos países de Latinoamérica. ORCID ID: https://orcid.org/0000-0002-8783-9911.Email: sergio.sanchezfuentes@uam.es 


\section{David Jiménez Hernández}

Doctor en Ciencias Sociales y de la Comunicación por la Universidad Católica San Antonio de Murcia (UCAM), y Máster en Investigación e Innovación en Infantil y Primaria por la Universidad de Murcia. Actualemte trabaja como ayudante doctor en la UCAM, impartiendo docencia en los grados de Educación Infantil y Primaria y en el Máster de formación del profesorado. Sus líneas principales de investigación son los métodos didácticos de enseñanza activa, formación del professorado universitário, Tecnologías de la Información y la Comunicación en el aula. ORCID ID: https://orcid.org/0000-00021325-6609. Email: djimenez361@ucam.edu

\section{Patricia Sancho Requena}

Doctora en Psicología, con Máster en Psicología Clínica y Atención Sociosanitaria a la Dependencia, ha trabajado como docente en distintas universidades y departamentos y, actualmente, es profesora en el Departamento de Psicología Evolutiva y de la Educación de la Universitat de València. Como experta en Psicología Evolutiva y Metodología, centra su investigación en la aplicación de Modelo de Ecuaciones Estructurales al estudio del ciclo vital y la calidad de vida, y cuenta con numerosos artículos publicados en revistas peer review, algunos de ellos en primer cuartil. ORCID ID: http://orcid.org/0000-00028624-8757. Email: patricia.sancho@uv.es

\section{Irene Moreno-Medina}

Personal Docente Investigador en Formación del Departamento de Didáctica y Teoría de la Educación en la Universidad Autónoma de Madrid. Licenciada en Psicopedagogía y Diplomada en Educación Social (ambas por la Universidad de Málaga). Miembro de Grupo de Investigación "Cambio Educativo para la Justicia Social" (GICE), de la Universidad Autónoma de Madrid. Investigando sobre la cultura de los centros en contextos desafiantes. ORCID ID: https://orcid.org/OOOO-0002-3745-7253. Email: irene.morenomedina@predoc.uam.es 